\title{
Assembly of Aluminum Nanosheets Formed by Hydrothermal Synthesis
}

\author{
Haijiao Zhang, ${ }^{1}$ Huifang Xu, ${ }^{2}$ and Can $\mathrm{Zhao}^{1}$ \\ ${ }^{1}$ Modern Manufacture Engineering Center, Heilongjiang Institute of Science and Technology, Harbin 150027, China \\ ${ }^{2}$ School of Chemical Engineering and Technology, Harbin Institute of Technology, Harbin 150001, China
}

Correspondence should be addressed to Haijiao Zhang, seaboyfang@163.com and Huifang Xu, xuhf@hit.edu.cn

Received 27 May 2011; Revised 29 August 2011; Accepted 29 August 2011

Academic Editor: Ali Eftekhari

Copyright ( 2011 Haijiao Zhang et al. This is an open access article distributed under the Creative Commons Attribution License, which permits unrestricted use, distribution, and reproduction in any medium, provided the original work is properly cited.

\begin{abstract}
This work put forward a facile method for obtaining assembly of aluminum (Al) nanosheets on an $\mathrm{Al}$ substrate by hydrothermal process. The results revealed that the ammonia was crucial for forming the assembly of Al nanosheets. In addition, the morphology and microstructure of the as-prepared $\mathrm{Al}$ nanosheets were strongly dependent on the hydrothermal temperature. Further, the influence of surfactant on the morphology of $\mathrm{Al}$ nanostructures was discussed that the nanosheets could be obtained under CMC of Span-80. A mechanism for forming the assembly of Al nanostructures was proposed that the erosion process based on the vacancies and defects of $\mathrm{Al}$ film determined the morphologies of $\mathrm{Al}$ nanosheets according to the experimental results.
\end{abstract}

\section{Introduction}

Advanced nanostructured materials are of fundamental interests to chemistry and materials science [1-3]. Especially, nanostructured metal has aroused wide attention due to their unique optical, electrical, and magnetic properties, which are distinct from their bulk counterparts. Therefore, to design, pattern, and assemble them into functional threedimensional networks become significant challenges for practical nanodevices. Metallic aluminum ( $\mathrm{Al}$ ), as one of important metallic materials, has been widely used in physics, chemistry and biology. The flake $\mathrm{Al}$ as one of the most important metal pigments has been widely used in various fields, such as architecture, automotive, chemical industry, and printing. For the battery materials, the aluminum/air battery is one of the most important metal/air batteries, and especially suitable for being a power device. For these different applications, different-shaped nanostructures of $\mathrm{Al}$, such as nanoparticles [4-7], and nanowires [8], have been obtained by using a variety of synthesis systems. For example, Al nanowires have been obtained via a vapor-deposition method through the evaporation of Al films clearly exhibited a smaller lattice constant; and smaller thermal expansion coefficient compared with the Al film [9]. Compared with high-temperature techniques, low-temperature solu- tion reaction approaches are more attractive for their merits such as simplicity, commercial feasibility, and good potential for scale-up. Up to now, still few investigations were made on the effect of basic solution on metallic aluminum sheet and fabricated densely packed aluminum nanosheet as film on flat solid substrates.

In this letter, we described a facile method to prepare assembly of $\mathrm{Al}$ nanosheets directly onto the surface of $\mathrm{Al}$ foil under basic catalytic hydrothermal reaction. Our strategy to control the shape and orientation of crystallites consists of growing thin-film materials directly onto substrates. The results showed that ammonia was crucial for facilitating the formation of Al nanosheets, and the surfactant promoted the formation of regular nanosheets. The obtained assembly of $\mathrm{Al}$ nanosheets was promising for being used in the areas such as noise elimination and battery.

\section{Experiments}

A commercial aluminum slice, $0.3 \mathrm{~mm}$ in thickness, was sealed in vacuum tube and annealed at $500^{\circ} \mathrm{C}$ for an hour to remove organic impurities. The aqueous ammonia $\left(\mathrm{NH}_{3} \cdot \mathrm{H}_{2} \mathrm{O}\right)$, ethanol $\left(\mathrm{C}_{2} \mathrm{H}_{6} \mathrm{O}\right.$, i.e., EtOH $)$, and sorbitan monooleate $\left(\mathrm{C}_{24} \mathrm{H}_{44} \mathrm{O}_{6}\right.$, i.e., Span-80) were purchased from 
TABLE 1: Summarized reaction conditions of aluminum nanosheets.

\begin{tabular}{|c|c|c|c|c|c|c|c|c|}
\hline & \multirow{2}{*}{$\mathrm{NH}_{3} \cdot \mathrm{H}_{2} \mathrm{O}(\mathrm{mL})$} & \multirow{2}{*}{$\mathrm{EtOH}(\mathrm{mL})$} & \multirow{2}{*}{$\mathrm{H}_{2} \mathrm{O}(\mathrm{mL})$} & \multirow{2}{*}{ Span-80 (mL) } & \multicolumn{4}{|c|}{ Temperature/Hour $\left({ }^{\circ} \mathrm{C} / \mathrm{h}\right)$} \\
\hline & & & & & A & $\mathrm{B}$ & $\mathrm{C}$ & $\mathrm{D}$ \\
\hline $\mathrm{a}$ & \multirow{4}{*}{1.2} & \multirow{4}{*}{12} & 10 & 0 & \multirow{4}{*}{$120 / 24$} & \multirow{4}{*}{$140 / 24$} & \multirow{4}{*}{$160 / 24$} & \multirow{4}{*}{$180 / 12$} \\
\hline $\mathrm{b}$ & & & 9.9 & 0.1 & & & & \\
\hline c & & & 9.6 & 0.4 & & & & \\
\hline $\mathrm{d}$ & & & 9.2 & 0.8 & & & & \\
\hline
\end{tabular}

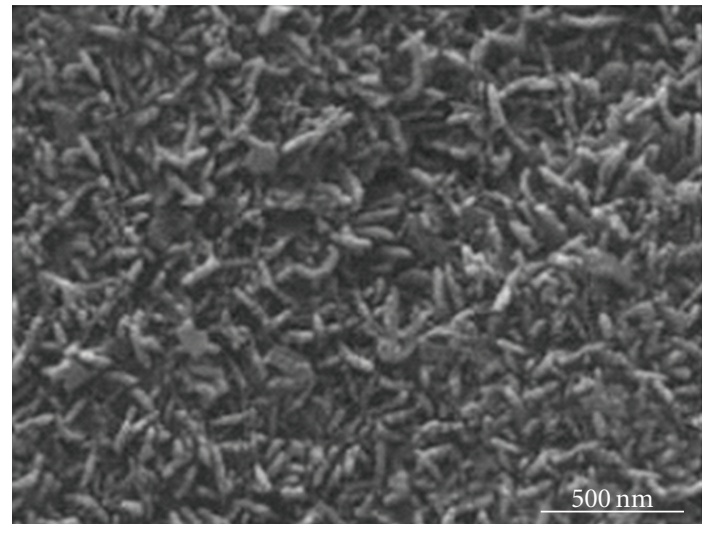

(a)

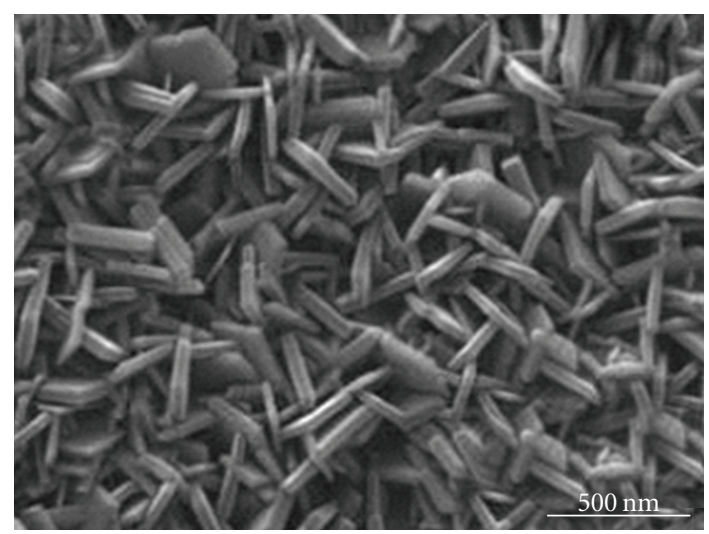

(c)

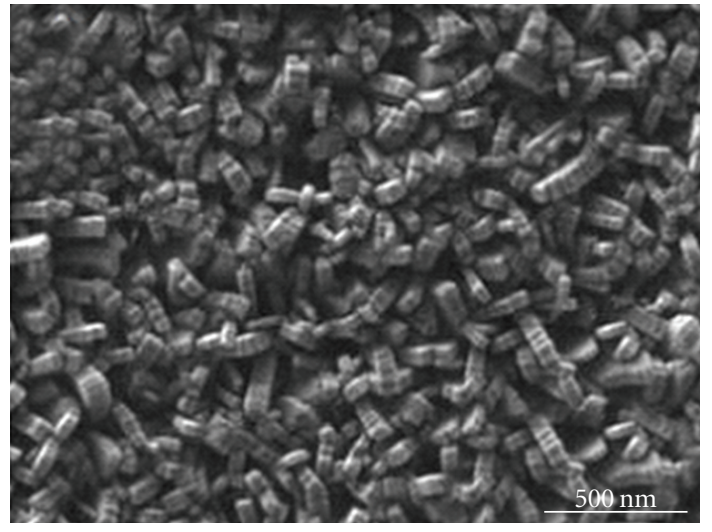

(b)

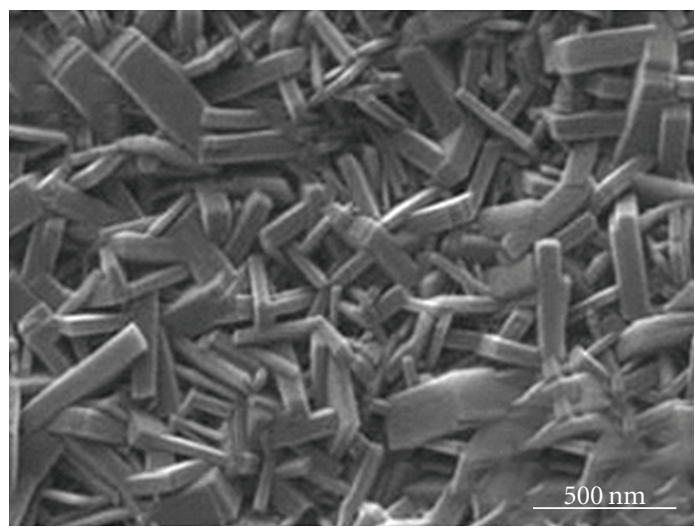

(d)

Figure 1: SEM images of $\mathrm{Al}$ nanosheets prepared at different hydrothermal temperatures (a) $120^{\circ} \mathrm{C}$, (b) $140^{\circ} \mathrm{C},(\mathrm{c}) 160^{\circ} \mathrm{C},(\mathrm{d}) 180^{\circ} \mathrm{C}$.

Tianjin Kermel Chem. Rea. Co, Ltd. and used as received. First, the treated aluminum slice was washed by ethanol and acetone several times, and consequently dried in flowing nitrogen atmosphere. Following that, a piece of aluminum about $10 \times 10 \mathrm{~mm}$ in size was added in the mixed solution including ammonia, ethanol, and Span-80 (the ratios of components were presented in Table 1). The mixed solution was stirred vigorously for $1 \mathrm{~h}$ and then put into a teflonlined stainless steel autoclave of capacity $50 \mathrm{~mL}$ up to $80 \%$ of the total volume. The autoclave was maintained at $120-180^{\circ} \mathrm{C}$ for $12-24 \mathrm{~h}$ and then cooled to room temperature. The obtained metallic foil was washed with distilled water and $\mathrm{EtOH}$ for several times and finally dried in vacuum at $60^{\circ} \mathrm{C}$ for $6 \mathrm{~h}$. The morphology images of the samples were recorded by scanning electron microscopy (HITACHI S-4300). Wide- angle X-ray diffraction (XRD, Rigaku-D/max- $\gamma$ pc instrument) was used to characterize the crystalline phase.

\section{Results and Discussion}

The synthesis parameters for obtaining the nanostructures of metal $\mathrm{Al}$ are listed in Table 1 . The ratios between $\mathrm{H}_{2} \mathrm{O}$ and Span-80 were changed for different samples.

The typical SEM images of products prepared without Span-80 are shown in Figure 1. It is found that the wormlike nanosheets were obtained with a typical width of $25 \mathrm{~nm}$ and a length of up to $100 \mathrm{~nm}$ by the hydrothermal reaction at $120^{\circ} \mathrm{C}$ (Figure $1(\mathrm{a})$ ). however, the rectangular nanostructures were found with sides measuring of $50-500 \mathrm{~nm}$ and a thickness of $20-50 \mathrm{~nm}$ when the reaction temperature was in 


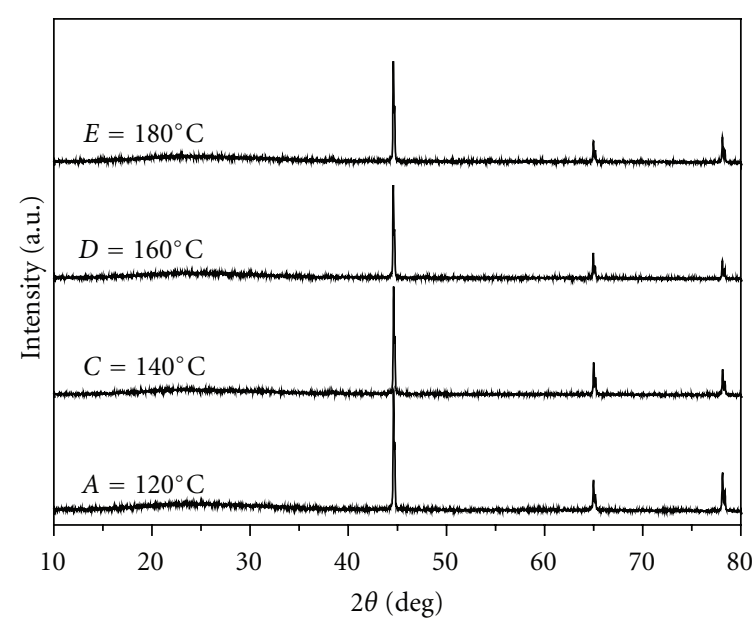

(a)

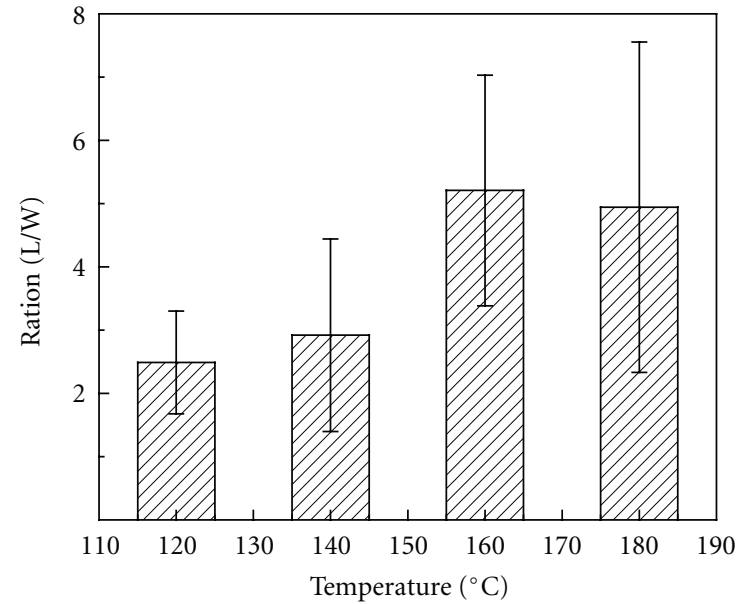

(b)

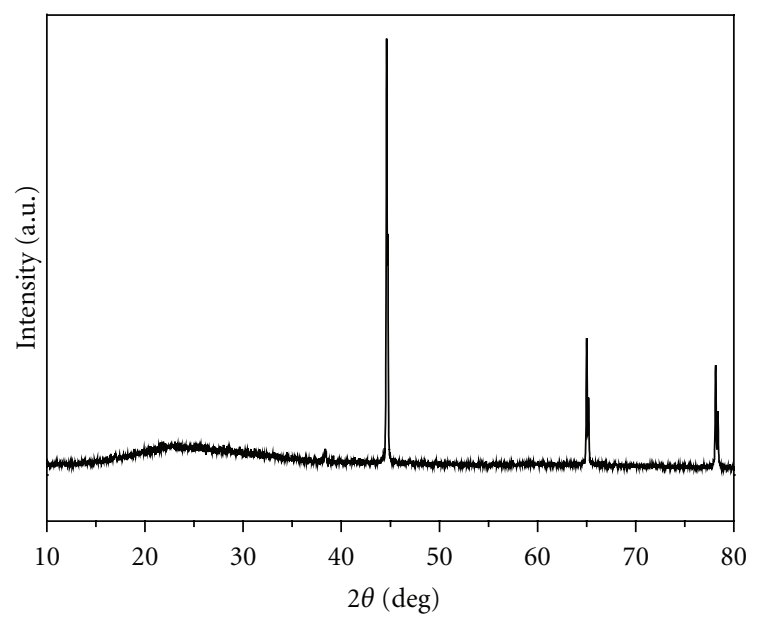

(c)

FIGURE 2: XRD patterns (a), the ratios of length to width for $\mathrm{Al}$ nanosheets prepared at different hydrothermal temperatures (b), and XRD pattern of Al substrate starting materials (c).

the range from 140 to $180^{\circ} \mathrm{C}$ (Figures $1(\mathrm{~b})$ to $1(\mathrm{~d})$ ). That is, the average thickness of the prepared aluminum nanosheets at $140^{\circ} \mathrm{C}, 160^{\circ} \mathrm{C}$, and $180^{\circ} \mathrm{C}$ was $20 \mathrm{~nm}, 35 \mathrm{~nm}$ and $50 \mathrm{~nm}$, respectively. Additionally, the samples synthesized at $160^{\circ} \mathrm{C}$ showed more regular nanosheet structures than other samples. These results suggested that the hydrothermal temperature played a crucial role on the morphologies of $\mathrm{Al}$ nanosheets. Further, the $\mathrm{Al}$ nanosheets prepared at high hydrothermal temperature showed the smooth surfaces, and the width of nanosheets was increased with increasing the hydrothermal temperature. Therefore, it is predicated that the thickness and the surface morphology of the $\mathrm{Al}$ nanosheets could be simply tailored by adjusting the hydrothermal temperature.

Figure 2(a) reveals the XRD patterns of the as-deposited $\mathrm{Al}$ nanostructures, confirming the crystalline structure of tetragonal Al. The XRD patterns were well consistent with JCPDS card no. 04-0787. It should be noted that there were no peaks attributable to aluminum oxide, indicating that these $\mathrm{Al}$ nanostructures were free of the commonly encountered oxidation that occurred in many preparation processes. The different ratios between length and width of products were shown in Figure 2(b). It could be found that the products synthesized at $160^{\circ} \mathrm{C}$ exhibited a higher ratio than other samples. This result was consistent with the conclusions obtained from Figure 1. It is also to be noted on the other hand that the increase in length size of the alumina particles was not so prominent but the increase in width was obvious with increasing the hydrothermal temperature, probably indicating that with increasing surfactant concentration above CMC, there was no significant increase in the size of the "water pool" to affect the surfaces of aluminum sheets [9].

These results reveal that the surface nanostructures of $\mathrm{Al}$ foil could be controlled by adjusting hydrothermal reaction temperature under basic conditions. Additionally, the surfactant of Span-80 also played an important role in modifying the nanostructures of Al foils. Figures 3(a) to 3(c) showed the SEM images of different $\mathrm{Al}$ nanostructures synthesized at $140^{\circ} \mathrm{C}$ with various concentrations of Span-80. It was found 


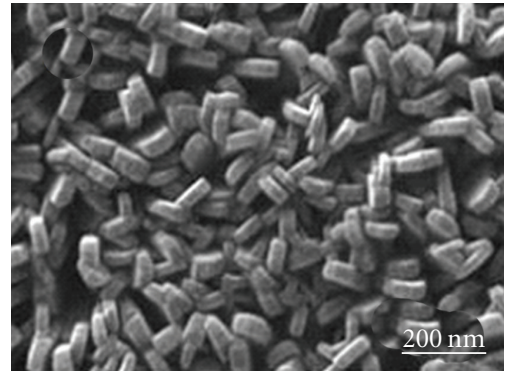

(a)

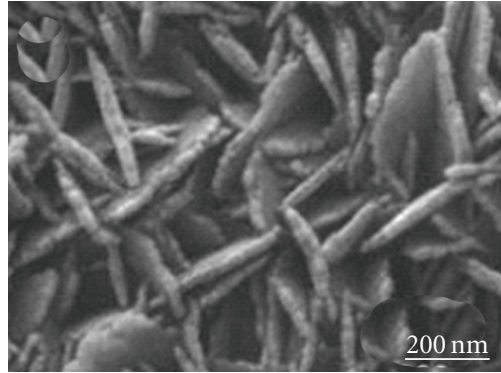

(b)

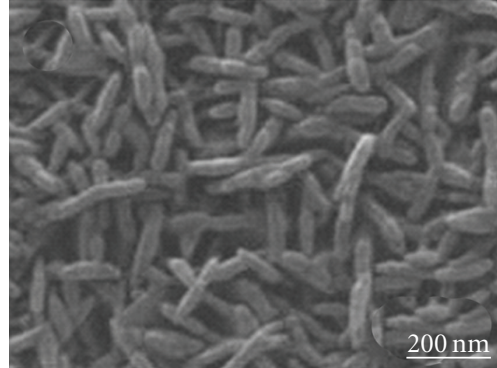

(c)

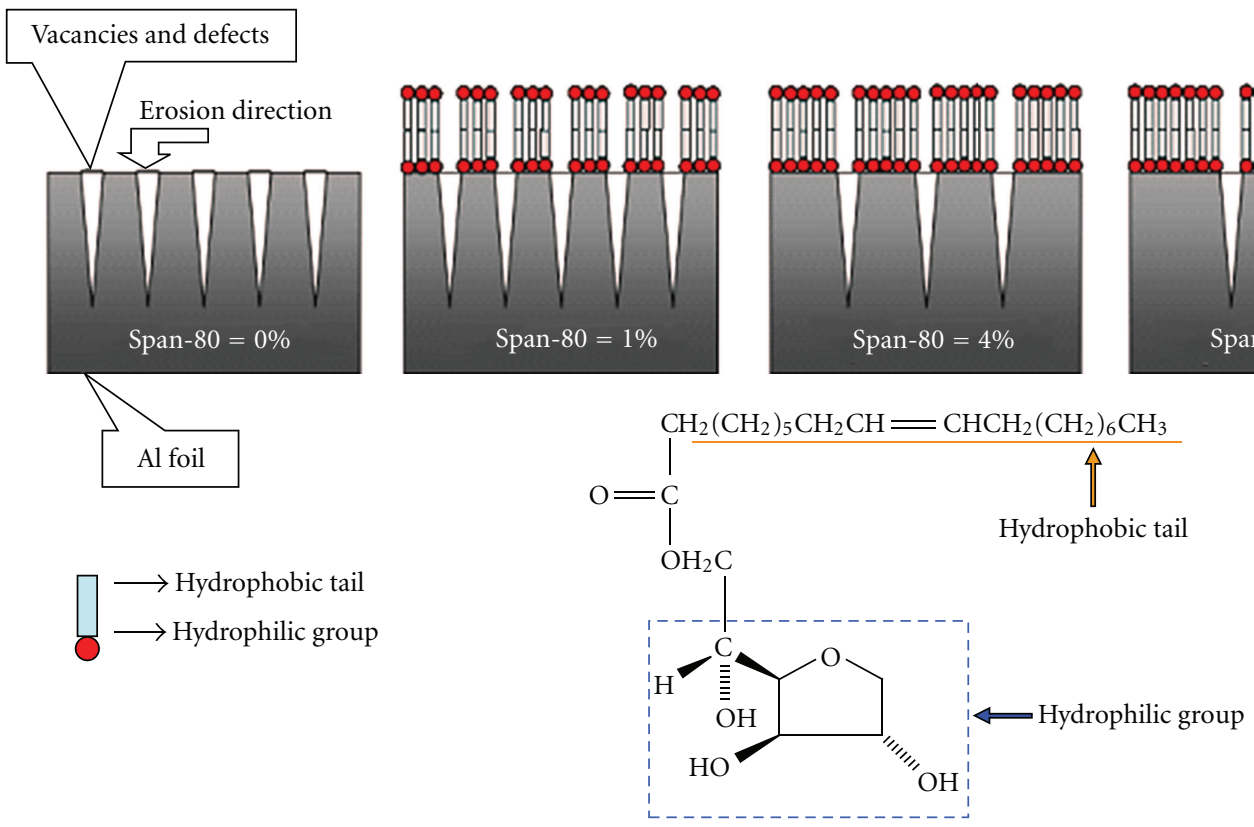

(d)

FIGURE 3: SEM images of Al nanosheets prepared with different concentrations of Span-80 (a) 1\%, (b) 4\%, (c) 8\% (Vol.\%), and (d) schematic illustration of forming $\mathrm{Al}$ nanostructures by hydrothermal method.

that the length of $\mathrm{Al}$ nanosheets was increased with increasing the concentration of Span-80 from 1\% to 8\% (Vol.\%). In addition, the morphologies were changed from smooth rectangular to coarse nanosheets. Probably, this occurred due to insufficient decrease in water-oil interfacial tension and correspondingly, incomplete break down of the sol droplets. But the breakdown of sol droplets to nanometric sizes was also affected by an optimized amount of the surfactant [9]. These results may be related to the molecular structure of Span-80. The formation process of $\mathrm{Al}$ nanostructures was proposed in Figure 3(d). As shown in Figure 3(d), lots of vacancies and defects were existed on the surface of $\mathrm{Al}$ foil. During the hydrothermal reaction, the erosion process was occurred under basic condition when there was no Span80 in the system. When the surfactant was added, as the $\mathrm{Al}-\mathrm{O}$ groups on the surface of $\mathrm{Al}$ foil exhibited hydrophilic properties, the hydrophilic groups of Span- 80 could be adsorbed on the surface of $\mathrm{Al}$ substrate by the electrostatic attraction. Then, a thin protective film was formed. The surface of $\mathrm{Al}$ foil could be further wetted and corroded by the mixed solution with increasing the concentration of
Span-80, which accelerated the erosion of Al film. Then, the thin $\mathrm{Al}$ nanosheets were synthesized and the ratio of width to thickness was increased. In addition, the ammonia was another key factor to influence the morphology of products. In this experiment, the addition of ammonia accelerated the erosion process of $\mathrm{Al}$ substrate. Also, the concentration of Span-80 limited the reaction between $\mathrm{Al}$ substrate and ammonia. Therefore, the nanosheets were synthesized by increasing the concentration of Span-80.

\section{Conclusions}

In this paper, we discussed a facile method for obtaining assembly of aluminum ( $\mathrm{Al}$ ) nanosheets on an $\mathrm{Al}$ substrate by hydrothermal process. The results showed that the morphology and microstructure of the as-prepared $\mathrm{Al}$ nanosheets were strongly dependent on the reaction factors. Ammonia was a crucial factor for formation of the nanosheets. In addition, the morphology of $\mathrm{Al}$ nanosheets was strongly depended on surfactant of Span-80. Further, a mechanism for forming the assembly of aluminum nanosheets was 
proposed. The approach presented here opened a new light in the application of hydrothermal method in preparing metal nanostructures, and the formed assembly of Al nanostructures are promising to be used in the fields of physics, chemistry, and biology.

\section{Acknowledgment}

This work was supported by the NSF of China (51003020).

\section{References}

[1] Y. J. Hsu and S. Y. Lu, "Preparation of Nanosized ZnSPassivated CdS Particle Films via the MOCVD Process with Cofed Single Source Precursors," Langmuir, vol. 20, no. 1, pp. 194 201, 2004.

[2] M. -H. Zhao, Z. -L. Wang, and S. X. Mao, "Piezoelectric characterization individual zinc oxide nanobelt probed by piezoresponse force microscope," Nano Letters, vol. 4, no. 4, pp. 587590, 2004.

[3] S. Sun, C. B. Murray, D. Weller, L. Folks, and A. Moser, "Monodisperse FePt nanoparticles and ferromagnetic FePt nanocrystal superlattices," Science, vol. 287, no. 5460, pp. 1989-1992, 2000.

[4] E. Stratakis, M. Barberoglou, C. Fotakis, G. Viau, C. Garcia, and G. A. Shafeev, "Generation of al nanoparticles via ablation of bulk al in liquids with short laser pulses," Optics Express, vol. 17, no. 15, pp. 12650-12659, 2009.

[5] E. Stratakis, V. Zorba, M. Barberoglou, C. Fotakis, and G. A. Shafeev, "Laser writing of nanostructures on bulk $\mathrm{Al}$ via its ablation in liquids," Nanotechnology, vol. 20, no. 10, Article ID 105303, 2009.

[6] G. Viau, V. Collière, L. M. Lacroix, and G. A. Shafeev, "Internal structure of Al hollow nanoparticles generated by laser ablation in liquid ethanol," Chemical Physics Letters, vol. 501, no. 4-6, pp. 419-422, 2011.

[7] P. Sen, J. Ghosh, A. Abdullah, P. Kumar, and Vandana, "Preparation of $\mathrm{Cu}, \mathrm{Ag}, \mathrm{Fe}$ and $\mathrm{Al}$ nanoparticles by the exploding wire technique," Proceedings of the Indian Academy of Sciences: Chemical Sciences, vol. 115, no. 5-6, pp. 499-508, 2003.

[8] L. Li, X. Xu, H. Chew et al., "Direct growth of Al nanowire arrays: thermal expansion and field emission properties," Journal of Physical Chemistry C, vol. 112, no. 14, pp. 5328-5332, 2008.

[9] B. Siladitya, M. Chatterjee, and D. Ganguli, "Role of a surface active agent in the sol-emulsion-gel synthesis of spherical alumina powders," Journal of Sol-Gel Science and Technology, vol. 15 , no. 3, pp. 271-277, 1999. 

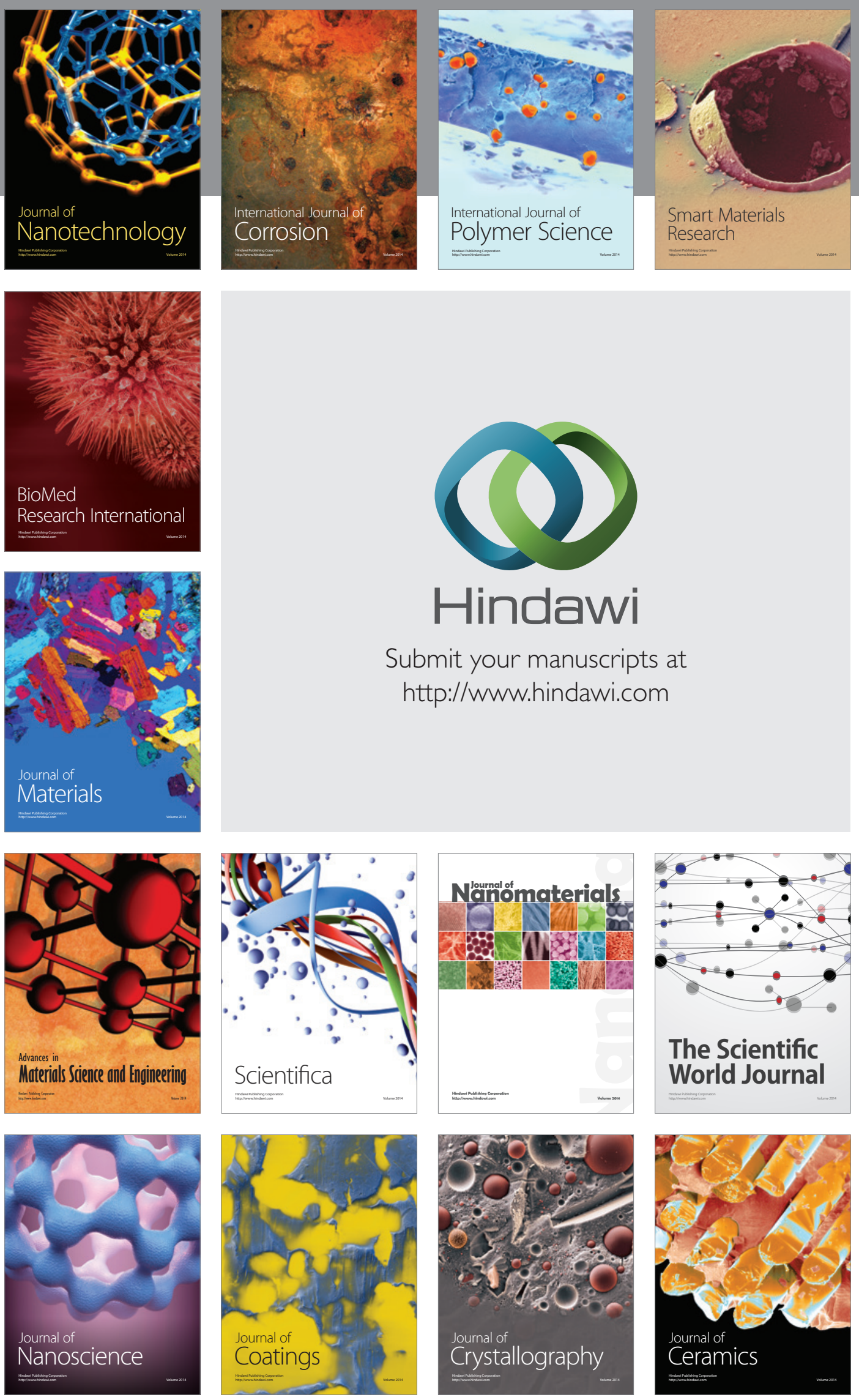

The Scientific World Journal

Submit your manuscripts at

http://www.hindawi.com

\section{World Journal}

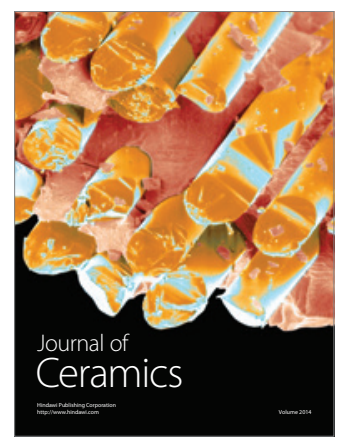

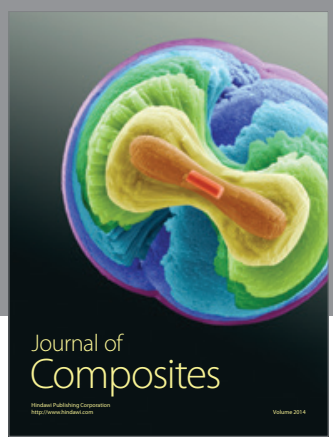
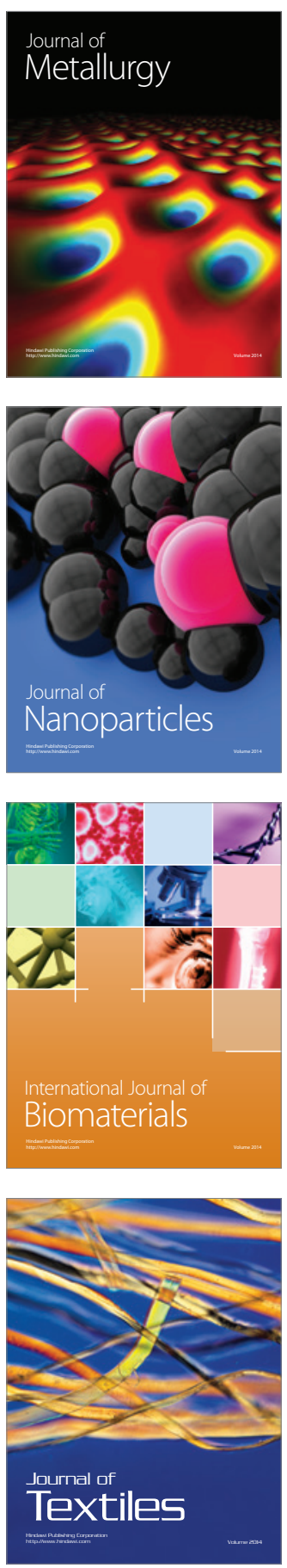